\title{
Correspondence
}

\section{Commissions, coercion and choice}

The recent letter by Das ${ }^{1}$ was a succinct and clear appraisal of much of the Schizophrenia Commission's report. Das takes issue with the report's point that 'shared decision making on medication choices is essential', commenting that this cannot refer to 'the patient with chronic schizophrenia who refuses all medication' and that it is 'obvious to any psychiatrist that the report is not referring to this type of patient'.

I do not think the issue is as cut and dried within psychiatry as Das suggests. A recent editorial in the British Journal of Psychiatry ${ }^{2}$ which proposed that patient choice should indeed be extended to patients with psychotic disorders was met with nothing but nodding heads and commendations. However, the reality of journal pages and that of NHS psychiatry is often as distinct as that of the psychiatrist and the patient with psychosis. The way decisions concerning antipsychotic medication are made is frequently quite straightforward - if you have a chronic psychotic illness and refuse treatment, coercion will be used to ensure you are receiving medication.

If two parties have diametrically opposed views and are unable to compromise, then shared decision-making will be limited. We feel ethically justified in coercive treatment because we have decided that the patient does not have the capacity to decide on their treatment and therefore we are acting in their best interests. This highlights the two issues that are at the heart of the debate: 'capacity' and 'best interests'.

In practice, the judgement of capacity is often treated as a relatively simple matter, where, if a patient is refusing medication, they are seen as lacking the ability to weigh the information necessary to make this decision. The question of best interests is also frequently treated as uncomplicated, namely that it is in a patient's best interests to ensure they receive antipsychotic medication. But can we say we know what the best interests are for a patient who has spent the majority of their life trying to avoid contact with psychiatry and has personally felt no benefit from antipsychotic medication? Are we justified in saying, as Das does, that they were 'rescued from [ ... ] a salubrious itinerant lifestyle'? I do not think it is an impossible notion that an individual might prefer to live a withdrawn or chaotic life rather than one imposed on them externally.

Putting yourself in a patient's shoes to determine their best interests means understanding their wishes and motivations, not those of the medical profession or the rest of society. When a psychiatrist judges the best interests of a patient, the decision is formed from within a system that is extremely risk averse and often appears to be built on the premise of avoiding psychosis to the exception of all else. The scales we use to weigh various eventualities (e.g. psychosis, stability, side-effects, risk to others) may be quite different to those that individuals would choose for themselves in their pre-morbid state. Best interests for an individual will vary tremendously depending on the attitude that person has to life. Antipsychotics can be tremendously beneficial but there will be a subset of patients who receive little benefit from them. Research on treatment trajectories ${ }^{3}$ supports the idea that the benefit of antipsychotic medication may primarily be in 'dramatic responders', with patients who show a more modest response being difficult to distinguish from those treated with placebo. Furthermore, as in all of medicine, there will be patients whose long-term outcome would have been superior without treatment. Despite best intentions, our interventions may sometimes harm patients and recognising this is the only way we can make progress in minimising this unpalatable truth.

The problems we have to deal with are not easy to solve. Terms such as 'patient choice' are meaningless and obscure the issues at heart. We need to talk about specifics. Many of the decisions we deal with as psychiatrists are made on the basis of limited evidence and cannot be made objectively. It aids both us and our patients to realise that although psychiatric training and experience opens one's eyes to many of the complexities of mental illness, it can just as easily narrow one's view as to how a life can be lived.

1 Das S. Critical appraisal of the Schizophrenia Commission's report. Psychiatrist 2013; 37: 72-3.

2 Morrison AP, Hutton P, Shiers D, Turkington D. Antipsychotics: is it time to introduce patient choice? Br J Psychiatry 2012; 201: 83-4.

3 Marques TR, Arenovich T, Agid O, Sajeev G, Muthen B, Chen L, et al. The different trajectories of antipsychotic response: antipsychotics versus placebo. Psychol Med 2011; 41: 1481-8.

Robert A. McCutcheon, CT1 in psychiatry, Oxleas NHS Foundation Trust, London, UK, email: robert.mccutcheon@kcl.ac.uk

doi: $10.1192 / p b .37 .5 .179$

\section{How does liothyronine prevent ECT-induced memory impairment?}

In Masoudzadeh et al's $s^{1}$ evaluation of the use of liothyronine as an adjunct to electroconvulsive therapy (ECT), the finding of improved outcome on both the Hamilton Rating Scale for Depression and the revised Wechsler Memory Scale is encouraging, especially in view of the relatively small sample size.

The authors postulate that liothyronine augmentation might achieve improved cognitive performance by suppressing thyrotropin releasing hormone (TRH). As TRH is an anticonvulsant, decreased production might result in a lowered seizure threshold and consequently a lower stimulus dose during ECT. It would be of interest to know whether the authors found a reduction of seizure threshold in the liothyronine group when compared with the placebo group; such a finding would lend support to the proposed mechanism of action.

Depression is associated with cognitive impairment. ${ }^{2}$ The better cognitive function achieved in the liothyronine group may have been a consequence of the greater improvements in depression seen in this group rather than a consequence of TRH suppression and reduced seizure threshold. Liothyronine has been shown to improve the outcome of depression when used as an augmentation to standard antidepressant therapy ${ }^{3}$ and it is possible that similar 
improvements in efficacy are observed when liothyronine is used concurrently with ECT. An analysis of the results that allows for control of the confounding effects of the severity of depression may be helpful, but in a small study there is an increased risk of type II error.

Many of the cognitive effects of ECT tend to normalise over time ${ }^{4}$ and a longer-term study is required to fully assess the effects on cognition of liothyronine augmentation. Such a study might also allow the severity of depression in both groups to equalise, such that an assessment of cognitive function independent of the severity of depression is possible. Notwithstanding these limitations, the work by Masoudzadeh and colleagues is a welcome addition to the literature, although it is the improved outcome in depression achieved by the addition of liothyronine that might turn out to be their most significant finding.
1 Masoudzadeh A, Yahyavi ST, Rashidi H, Mohammadpour RA, Kiani R. Use of liothyronine in preventing electroconvulsive therapy-induced memory impairment: evaluation. Psychiatrist 2013; 37: 49-53.

2 Austin M-P, Mitchell P, Goodwin GM. Cognitive deficits in depression. Possible implications for functional neuropathology. Br J Psychiatry 2001; 178: $200-6$.

3 Joffe RT, Singer W, Levitt AJ, MacDonald C. A placebo-controlled comparison of lithium and triiodothyronine augmentation of tricyclic antidepressants in unipolar refractory depression. Arch Gen Psychiatry 1993: 50: 387-93.

4 Sackeim HA, Prudic J, Fuller R, Keilp J, Lavori PW, Olfson M. The cognitive effects of electroconvulsive therapy in community settings. Neuropsychopharmacology 2007; 32: 244-54.

Tim N. Oakley, lead consultant for ECT, Northumberland, Tyne and Wear NHS Foundation Trust, George's Park, Morpeth, UK, email: tim.oakley@ntw.nhs.uk

doi: 10.1192/pb.37.5.179a 